\title{
Du référendum praecox à un abortus ex utero, ou comment le Phénix pourrait renaître de ses cendres
}

Un conseiller des Etats par ailleurs contestable de Suisse centrale n'a pas manqué récemment de nous sermonner vertement et de parler de référendum praecox. Ce faux pas rhétorique allait toutefois dans la bonne direction: le mot praecox associé généralement en clinique à un trouble des fonctions génitales - convient parfaitement à la $2^{\mathrm{e}}$ révision de la LAMal dans son ensemble. Le Conseil national vient d'éviter au peuple suisse d'infliger une correction à son rejeton. L'abortus ex utero du 17 décembre 2003 était la solution la plus aisée.

Seule organisation à le faire au bon moment, le 11 octobre 2003, la FMH s'est on ne peut plus clairement prononcée contre cette fausse couche légale. Cette dernière ne résolvait aucun problème, elle en aurait aussi créé d'autres. Et ceci à l'heure où beaucoup ne faisaient qu'en peser le pour et le contre. La clarté de la position de la FMH a contribué de façon décisive à anéantir ce projet désastreux qui, aux yeux de nombreux parlementaires, aurait de toute façon été jeté aux orties par le peuple. Empêcher un référendum inutile: une contribution substantielle du corps médical à la diminution des coûts de la santé.

Un arrière-goût amer demeure: pourquoi a-t-on cherché à introduire en contrebande cette révision tout en nourrissant le peuple suisse de belles paroles? La FMH est-elle en passe de devenir une donneuse de leçons à la classe politique? Un tel rôle ne saurait vraiment la satisfaire. Notre devoir de médecin est de traiter les patients et de promouvoir un approvisionnement médical raisonnable - et non de faire d'une politique partisane notre tâche journalière. Nous ne voulons pas devoir une nouvelle fois jouer les avocats du peuple et remettre sur le bon chemin les dérives politiques.

Ceux qui, dans nos cercles, croiraient maintenant pouvoir se reposer et renouer avec leur train-train quotidien seraient l'objet d'une hallucination fatale. Le succès impose ses contraintes. Nous serons sollicités de toutes parts - à raison, sans doute - pour présenter nos propres propositions d'un système de santé suisse et apporter des réponses à des questions aussi brûlantes que l'augmentation des primes, l'accroissement de l'efficacité de l'approvisionnement médical, la sécurité de nos traitements, le rationnement de nos prestations, etc. Une fois de plus, il faudra réunir toutes nos forces pour trouver des réponses, d'autant plus que la controverse sur maintes de ces questions demeure vivace dans nos rangs.

Notre action sera déterminante, elle est aussi le fondement de notre crédibilité. Le TARMED est notre hic Rhodus, hic salta, il nous met en demeure de montrer sans attendre ce dont nous sommes capables. Pour toutes les questions encore à résoudre - tarif des médecins agréés, amélioration de la structure tarifaire pour les disciplines opératoires - les négociations intelligentes et compétentes des sociétés cantonales de médecine et de la FMH ont créé des conditions générales propices à l'introduction du TARMED dans le domaine LAMal ambulatoire. Les problèmes qui viennent de voir le jour dans le secteur ambulatoire hospitalier montrent à l'évidence ce à quoi peuvent conduire des conditions-cadres défavorables et des négociations sans juste appréciation de la situation.

Dans les mois qui suivront, l'opinion publique ne manquera pas d'observer très attentivement les positions que prendra la FMH sur les questions de politique de santé et de voir si elle parvient à mettre en pratique le TARMED dans sa sphère d'influence. Si nous devions à nouveau sombrer dans l'orgueil et céder à l'arrogance, le fœtus avorté ne pourrait, tel le Phénix, que renaître de ses cendres. Un Phénix terrifiant, pour lequel le référendum ne serait même plus une menace.

Hans Heinrich Brunner, président de la FMH 